\title{
ESPÁTULA PUPILÔMETRO: \\ UM INSTRUMENTO PARA MEDIDA DO DIÂMETRO PUPILAR
}

\author{
Regina Marcia Cardoso de Sousa * \\ Maria Sumie Koizume **
}

RESUMO - As autoras apresentam instrumento para medida do diâmetro pupilar, constituído de 9 círculos pretos impressos em escala crescente, numa espátula de madeira, com diâmetro mínimo de $1 \mathrm{~mm}$ e máximo de $9 \mathrm{~mm}$. Para este estudo chamam o aparelho de espátula de pupilômetro e foi usado por 180 enfermeiros para verificar a acuidade destes na leitura dos círculos milimétricos e a efetividade do uso deste aparelho. Os resultados mostraram que a espátula de pupilômetro provê forma objetiva para a mensuração pupilar, inócua ao indivíduo examinado e como instrumento de baixo custo e fácil manuseio.

ABSTRACT - The authors show an advice for checking the pupillary diameter, which was made up of a 9 black printed circles, progressively larger, with a minimum diameter of $1 \mathrm{~mm}$ and a maximun of $9 \mathrm{~mm}$, affixed to wooden tongue depressor. For this study they call this gauge "Pupillometer Tongue Depressor" and it has been checked out by 180 burses for the reading accuracy of the millimeter circles with the "Pupillometer Tongue Depressor" and the effectiveness of this tool as a pupillary diameter gauge. Results yeld information that the "Pupillometer Tongue Depressor" provide an objective form for pupillary measurement, innocuous to the checked individual and an instrument of low cost and easy handling.

\section{INTRODUÇĀO}

A necessidade de obter-se dados precisos no exame físico de enfermagem, tem propiciado o aprimoramento de instrumentos que permitem ao enfermeiro obter dados objetivos acerca de indivíduo alvo de sua assistência.

A coleta e registro de um dado de forma objetiva é importante não só para uma avaliação isolada, como tambem, para compor o todo do paciente ou possibilitar avaliações sistemáticas seqüenciais do mesmo.

Dentro da avaliação neurológica, um dos parâmetros que tem sido largamento empregado, é o exame do tamanho pupilar. Algumas opções jâ são encontradas na prática dos enfermeiros que permitem obter esse dado de forma simples e segura ao paciente.

Entre essas opções, uma das formas empregadas é a medida comparativa da pupila, realizada através do uso de um instrumento elaborado com uma espátula de madeira e uma escala de círculos progressivamente maiores em $1 \mathrm{~mm}$.

Esta espátula adaptada, já descrita por LORD-FEROLI, MAGUIRE-McGINTY ${ }^{10}$ e comentada por PASCOE ${ }^{16}$, foi modificada pelas autoras deste estudo e denominada "Espátula
Pupilômetro" (E.P.). Este instrumento foi então testado, visando basicamente obter-se dados que subsidiem a implementaçao do seu uso.

São portanto objetivos do presente estudo:

- determinar a precisão da leitura de círculos milimetrados impressos em cartão, utilizando a E.P.;

- validar a efetividade da medida do diâmetro pupilar com o uso da E.P. por meio de cotejamento dos valores obtidos por pares de enfermeiros;

- verificar as dificuldades sentidas pelos enfermeiros na medida do diâmetro pupilar com o uso da E.P..

\section{MATERIAL E MÉTODO}

A população deste estudo foi constiturda de 180 enfermeiros, de 6 hospitais do munićpio de São Paulo, sendo 5 destes, entidades da rede pública e um da rede privada.

Fizeram parte deste estudo enfermeiros com experiência na assistência a paciente graves, que concordaram em participar, sendo exclurdos os que referiram dificuldades para fazer leitura

\footnotetext{
* Professor Assistente do Departamento de Enfermagem Médico-Cirúrgicá da Escola de Enfermagem da Universidade de São Paulo

** Prof essor Assistente Doutor do Departamento de Enfermagem Médico-Cirúrgica da Escola de Enfermagem da Universidade de São Paulo
} 
próxima e aqueles hipermétropes ou presbíopes diagnosticados, sem lentes corretivas no momento da coleta de dados.

A avaliação do diâmetro pupilar foi realizada em 48 pacientes, sendo critério para sua escolha, terem como diagnóstico uma afecção neurológica ou apresentarem alteração de nível de consciência consequiente à sua afecção de base ou intercorrência no curso da doen$\mathrm{Ça}^{10},{ }^{17},{ }^{18}$. Dentre os pacientes que atenderam a este critério, foram exclurdos aqueles com edema palpebral acentuado, nos quais encontravase impossibilitada a exposição completa da pupila, e os que apresentavam manchas ou ferimentos em esclerótica, íris e pupila, que dificultavam a execução da medida do diâmetro pupilar.

Para a coleta dos dados foram utilizados os seguintes instrumentos: "Espátula Pupilômetro" (E.P.); "Fichas-teste" e folhas de registro de dados dos enfermeiros e pacientes.

A E.P. (ANEXO 1) testada neste estudo é uma espátula de madeira, na qual foi afixada, em ambos os lados, uma escala em plano vertical de círculos pretos de 1 a $9 \mathrm{~mm}$.

Esse instrumento $\varepsilon$ semelhante ao utilizado por LORD-FEROLI, MAGUIRE-McGINTY ${ }^{10}$, sendo que a principal diferença reside na fixação de escalas nas duas faces da espátula, com círculos dispostos de forma a possibilitar maior aproximação às pupilas, tanto no olho direito como esquerdo (ANEXO 1).

Embora a E.P. já tivesse sido testada por LORD-FEROLI, MAGUIRRE-McGINTY ${ }^{10}$, não foi estabelecido em seu estudo a origem e tipo de diferenças mais frequientemente encontradas nos valores obtidos nas medidas realizadas com esse instrumento, dados que permitem delinear o quanto os números obtidos nas medidas realizadas com a E.P. expressam o valor da grandeza estudada.

Foram construídas então, 900 "Fichas-teste" de 15 tipos diferentes. Nesses cartōes foram impressos: os dados para recuperar quem fez aquele teste, uma letra código de identificação dos diâmetros dos círculos e dois círculos paralelos (ANEXO 2).

Os 15 tipos diferentes de "Fichas-teste", distinguiram-se entre si pelas diferenças dos diâmetros, os quais foram aleatoriamente escolhidos e pelas letras de identificação, que permitiam às pesquisadoras reconhecer de imediato o valor preciso dos círculos desenhados.

Entre os 15 diferentes tipos de fichas apresentadas aos enfermeiros, em 9 tipos, encontravam-se dois círculos de diâmetros iguais (fichas com cérculos de diâmetros simétricos) e em 6 tipos, dois círculos de diâmetros diferentes (fichas com círculos de diâmetros assimétricos).

Os 180 enfermeiros testaram a E.P. em duas etapas de coleta de dados. Na primeira etapa, quando verificaram com a E.P. o diâmetro de círculos desenhados em "Fichas-teste" e na segunda etapa, quando reunidos em pares verifisaram o diâmetro pupilar de um único paciente oreviamente selecionado.

Nessa segunda etapa, cada um dos elementos dos pares executou a medida independentemente, porem num intervalo de tempo inferior a cinco minutos, os dois enfermeiros realizaram a medida pupilar de um paciente previamente selecionado.

Antecedendo à execução da modida do diâmetro pupilar, os enfermeiros foram orientados quanto à técnica a ser executada e aos pacientes, sempre que possível, explicou-se a finalidade do estudo e solicitou-se sua participação.

\section{RESULTADOS E DISCUSSŌES}

Os 180 enfermeiros que testaram a E.P. neste estudo, caracterizaram-se por:

- apresentarem em sua maioria $(52,22 \%)$ alteração visual diagnosticada, sendo que entre estes a maioria fazia uso de lentes corretivas;

- $40 \%$ desses enfermeiros cursou sómente o tronco profissional comum da graduação, $41,11 \%$ cursou tambem habilitação ou especialização em Médico-Cirúrgica e 18,89\% eram habilitados ou especializados em outras áreas;

- o tempo de experiência profissional desses' enfermeiros foi bastante variável, havendo porém uma predominância de individuos na faixa de $1 \longmapsto 5$ anos em exerćcios na profissão;

- 52,78\% desses enfermeiros realizam a medida do diâmetro pupilar em suas atividades profissionais diárias, $42,22 \%$, verificam esporádicamente e $5,00 \%$ nunca verificam o diâmetro pupilar de pacientes

De acordo com a metodologia proposta os 180 enfermeiros preencheram um total um total de 900 "Fichas-teste", ou seja, registraram 1.800 valores nos círculos desenhados. Como conhecia-se o valor real da grandeza dos circulos desenhados, ao compará-lo ao valor registrado pode-se classificar as medidas como certas ou erradas. Nessa classificação considerouse como erro, a diferença entre o valor registrado e o verdadeiro valor da grandeza ${ }^{26}$, a auséncia de diferença entre esses valores e acertos. 
Tabela 1. Distribuição dos acertos e erros, dos registros das "Fichas-teste", segundo seu valor real e tipo de ficha. São Paulo, 1988.

\begin{tabular}{|c|c|c|c|c|c|c|c|c|c|c|c|c|c|}
\hline \multirow{2}{*}{$\begin{array}{l}\text { Valor } \\
\text { Real }\end{array}$} & \multicolumn{4}{|c|}{$\begin{array}{l}\text { Fichas com Círculos de } \\
\text { Diâmetros Simétricos }\end{array}$} & \multicolumn{2}{|c|}{$\begin{array}{l}\text { Subtotal } \\
\text { (1) }\end{array}$} & \multicolumn{4}{|c|}{$\begin{array}{l}\text { Fichas com Círculos de } \\
\text { Diâmetros Assimétrico }\end{array}$} & \multirow{2}{*}{\multicolumn{2}{|c|}{$\begin{array}{c}\text { Subtotal } \\
(2)\end{array}$}} & \multirow[t]{2}{*}{$\begin{array}{l}\text { Total } \\
\text { (1) e (2) }\end{array}$} \\
\hline & \multicolumn{2}{|c|}{ Acertos } & \multicolumn{2}{|c|}{ Erros } & № & 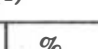 & A & rtos & No & os & 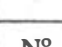 & & \\
\hline 1 & $\frac{N=}{120}$ & $\begin{array}{c}\% \\
11,15\end{array}$ & $\frac{N=}{-}$ & $\frac{\%}{-}$ & $\frac{N=}{120}$ & $\frac{\%}{11,15}$ & № & $\frac{\%}{-}$ & № & $\%$ & $\frac{1}{-}$ & $\%$ & 120 \\
\hline 2 & 120 & 11,15 & - & - & 120 & 11,15 & 60 & 8,29 & - & - & 60 & 8,29 & 180 \\
\hline 3 & 121 & 11,25 & 1 & 0,09 & 122 & 11,34 & 121 & 16,71 & - & - & 121 & 16,71 & 243 \\
\hline 4 & 121 & 11,25 & 1 & 0,09 & 122 & 11,34 & 119 & 16,44 & - & - & 119 & 16,44 & 241 \\
\hline 5 & 120 & 11,15 & - & - & 120 & 11,15 & 231 & 31,90 & 11 & 1,52 & 242 & 33,42 & 362 \\
\hline 6 & 109 & 10,13 & 11 & 1,02 & 120 & 11,15 & 111 & 15,33 & 11 & 1,52 & 122 & 16,85 & 242 \\
\hline 7 & 120 & 11,15 & - & - & 120 & 11,15 & - & - & - & - & - & - & 120 \\
\hline 8 & 104 & 9,67 & 16 & 1,48 & 120 & 11,15 & 46 & 6,35 & 14 & 1,94 & 60 & 8,29 & 180 \\
\hline 9 & 111 & 10,33 & 1 & 0,09 & 112 & 10,42 & - & - & - & - & - & - & 112 \\
\hline Total & 1046 & 97,23 & 30 & 2,77 & 1076 & 100,00 & 688 & 95,02 & 36 & 4,98 & 724 & 100,00 & 1800 \\
\hline
\end{tabular}

Observa-se na Tabela 1, que a distribuição dos erros e acertos ocorreu de forma semelhante nas fichas de círculos de diâmetros simetricos e assimetricos, já que em ambos os casos, a quase totalidade das medidas realizadas resultou em acertos $(97,23 \%$ e $95,02 \%)$ e a maioria $(2,77 \%$ e 4,98\%) em erros.

Nota-se porem, que os erros aconteceram numa maior frequiência quando as medidas foram realizadas em fichas com círculos de diâmetros assimétricos $(4,98 \%)$. A maior frequiência de erros nesse tipo de ficha, torna-se mais aparente quando verifica-se a proporção erro: acerto. Nas fichas com ćrculos de diâmetro assimétrico, esta proporção é de 1:19,11 (36:688), enquanto que nas fichas com diâmetros simetricos, é de 1:34,87 (30:1.046).

Tabela 2. Distribuição dos erros ocorridos nos 1.800 registros feitos nas "Fichas-teste" comparadas com o valor real. São Paulo, 1988

\begin{tabular}{c|c|c}
\hline Valor & Erro & $\begin{array}{c}\text { \% (em relação as } \\
\text { 1.800medidas) }\end{array}$ \\
\hline Real & No & 0,05 \\
\hline 3 & 1 & 0,05 \\
5 & 1 & 0,61 \\
6 & 11 & 1,22 \\
8 & 22 & 1,67 \\
9 & 30 & 0,05 \\
\hline Total & 1 & 3,65 \\
\hline
\end{tabular}

A Tabela 2 mostra que ocorreram 66 erros, entre as 1.800 medidas realizadas através da comparação entre círculos, utilizando-se instrumento em teste. A porcentagem de erros no conjunto de dados obtidos nessas medidas foi de $3,65 \%$, indicando que na sua quase totalidade, o resultado das medidas realizadas pelos 180 enfermeiros representou o verdadeiro valor da grandeza estudada.

Considerando-se que foram medidos círculos de 1 a $9 \mathrm{~mm}$ de diâmetro, verifica-se que os erros nunca oconeram quando o valor real do diâmetro do círculo era de 1,2 ou $7 \mathrm{~mm}$, aconteceram uma vez nos valores 3,4 e $9 \mathrm{~mm}$ e foram mais incidentes nos valores de 5, 6 e $8 \mathrm{~mm}$. Assim, constatou-se que os erros ocorreram de maneira mais expressiva entre as medidas realizadas em círculos de diâmetro de 5 ou mais milímetros.

A respeito dos erros, cumpre ainda mencionar, que todos ocorreram na amplitude de $1 \mathrm{~mm}$, variando seu sentido tanto para mais como para menos.

Após as medidas realizadas nas "Fichasteste", os enfermeiros, agrupados em pares, realizaram a medida do diâmetro pupilar de um paciente, previamente selecionado pelo pesquisador, conforme já descrito na metodologia.

O tempo medio gasto pelos enfermeiros, na execução dessa medida, foi de 43 segundos. A contagem desse tempo iniciou-se no momento em que o enfermeiro aproximou a espátula do olho D do paciente e foi encerrada somente após a medida e anotação no questionário do valor encontrado em ambos os olhos.

A verificação do diâmetro pupilar de pacientes realizada pelos 90 pares de enfermeiros, resultaram em 360 medidas (cada enfermeiro examinou as duas pupilas do paciente), as quais foram classificadas conforme a concordância obtida. Para essa classificação, analisou-se os valores das medidas realizadas por par de enfermeiros e considerou-se concordante aquelas mensuraçōes em que os valores obtidos foram iguais. 
Tabela 3. Distribuição dos valores das medidas do diâmetro pupilar segundo concordância obtida. São Paulo, 1988.

\begin{tabular}{c|c|c}
\hline Concordancia & № & $\%$ \\
\hline Sim & 212 & 58,89 \\
Não & 148 & 41,11 \\
\hline Total & 360 & 100,00 \\
\hline
\end{tabular}

A Tabela 3 mostra que a maioria das medidas pupilares realizadas pelos pares de enfermeiros resultaram em valores coincidentes $(58,89 \%)$. Todavia, uma grande parte destas mensuraçōes apontaram também para diferentes valores $(41,11 \%)$.

Embora concordância e acerto não possam ser considerados como sinonímia, é estabelecido uma relação entre eles, à medida que acertos, enquanto valores únicos, implicam na concordância. Porém, ao observar-se a Tabela 1, verifica-se que os índices de acertos $(97,23 \%$ e $95,02 \%$ ) encontram-se bem mais elevados que o de concordância de valores obtidos pelos pares de enfermeiros $(58,89 \%)$, como pode-se observar na Tabela 3.

Quando verificou-se a concordância, as pupilas humanas foram a grandeza estudada e dois novos fatores corroboraram no aparecimento de divergência nos valores encontrados. Por um lado há a possibilidade do diâmetro pupilar apresentar-se num valor intermediário da escala, levando a dúvidas quanto ao dígito que melhor representa sua grandeza e por outro, a "atividade da pupila", que acontece mesmo quando as condições do meio externo são mantidas constantes.

A pupila normal exibe movimentos pequenos e arrítmicos, aparentemente devido a estrmulos endógenos e são conhecidos como "agitação fisiológica" ou "atividades da pupila" ${ }^{2},{ }^{4},{ }^{8}$. Essas flutuações fisiológicas ocorrem de forma a proporcionar oscilações no orifício diafragmático das pupilas, superiores a $0,3 \mathrm{~mm}$ por segundo ${ }^{4}$, ocasionando assim constantes pequenas modificações no diâmetro pupilar.

Tabela 4. Distribuição dos valores das medidas discordantes do diâmetro pupilar, segundo diferença apresentada em milímetros. São Paulo, 1988.

\begin{tabular}{c|r|r}
\hline Diferenç em mm & № & $\%$ \\
\hline 1 & 142 & 95,95 \\
3 & 2 & 1,35 \\
2,70 \\
\hline Total & 4 & 100,00 \\
\hline
\end{tabular}

Pela Tabela 4, nota-se que na quase totalidade $(95,95 \%)$ das medidas em que ocorreu a discordância entre os resultados obtidos pelos pares, a diferença entre os valores indicados foi de $1 \mathrm{~mm}$.

Esta diferença apresenta a mesma amplitude encontrada nos erros ocorridos nas medidas dos círculos das "Fichas-teste", e é condizente com a intervenção nas mensurações, dos já mencionados fatores, que envolvem especificamente a medida pupilar.

Partindo-se para análise conjunta dos resultados apresentados nas Tabelas 3 e 4, observase que o diferencial de $1 \mathrm{~mm}$ ocorreu em grande parte das medidas realizadas, sendo entretanto, quase sempre esta, a diferença encontrada entre os valores obtidos.

Esse conjunto de resultados vem delinear a amplitude da incerteza da medida, em $1 \mathrm{~mm}$, valor este a ser considerado quando aplica-se os dados obtidos, nas mensurações realizadas utilizando-se a E.P..

As alterações de tamanho pupilar têm sido consideradas um importante sinal, que possibilita detectar anormalidades generalizadas ou localizadas no sistema nervoso ${ }^{10},{ }^{17},{ }^{18}$. No entanto, na análise desse dado, surgem alguns fatores que limitam a validade de medidas isoladas, tanto na fase de diagnóstico, como de seguimento do paciente, principalmente naqueles, em que busca-se detectar precocernente sinais de aumento da pressão intracraniana.

De maneira geral, as alterações na medida pupilar são circunscritas basicamente em duas categorias: as referentes as anomalias do tamanho (miose e midriase) e as apontadas em decorrência das diferenças entre as pupilas do indivíduo (anisocoria) 4 .

As anomalias referentes ao tamanho trazem algumas difuculdades para ser delineadas, a medida que existem várias interferências reconhecidas no diâmetro pupilar, tais como, emoções psíquicas ${ }^{2}$, excitaçōes sensiti$\operatorname{vas}^{3},{ }^{12},{ }^{21},{ }^{25}$, estado de vigniaa ${ }^{19}$ e outras, que nem sempre são possíveis de serem controladas. Desta forma, diferentes valores normais são descritos para o diâmetro pupilar ${ }^{2}$, 5 , encontrando-se assim uma extensa faixa de provável normalidade, que no entanto, apresenta, nos seus valores limites, possíveis sinais de alte ração.

Já ao analisar-se a desigualdade pupilar, encontram-se as possíveis assimetrias pupilares fisiologicas, que podem erroneamente ser con- 
sideradas como resultado de compressão do III nervo craniano ipsolateral à pupila de maior diâmetro ${ }^{17}$.

Desta forma, o tamanho pupilar, enquanto um item incluído e analisado dentro do exame neurologico ${ }^{1},{ }^{5},{ }^{6},{ }^{7},{ }^{9},{ }^{11},{ }^{13},{ }^{14},{ }^{15},{ }^{20},{ }^{22},{ }^{23},{ }^{24},{ }^{27}$, mostra sua maior expressividade quando suas alte- rações são interpretadas dentro do conjunto de dados complementares e evolutivos, onde o diferencial de $1 \mathrm{~mm}$, não pode significar por si só alterações ou anormalidades, mas sim, sinal de alerta que aponta para necessidade de avaliações complementares e mais frequientes, que confirmem ou não a existência de um processo de risco ao paciente.

Tabela 5. Distribuição dos pares de enfermeiros, segundo concordância nos valores obtidos na medida do diâmetro pupilar. São Paulo, 1988.

\begin{tabular}{c|c|c}
\hline Concordáncia na & No & \% \\
Medida & 41 & 45,55 \\
Em ambos os olhos & 24 & 26,67 \\
Em um dos olhos & 25 & 27,78 \\
Em nenhum dos olhos & 90 & 100,00 \\
\hline Total & & \\
\hline
\end{tabular}

Os resultados da Tabela 5 mostram que a maior parte dos enfermeiros $(45,55 \%)$ concordam com seus pares, no valor obtido na medida do diâmetro pupilar de ambos os olhos do paciente. Porém, observando-se ainda estes resultados, verifica-se que a maioria dos enfermeiros $(54,45 \%)$ não concordaram com o valor obtido em um ou ambos os olhos $(26,67 \%+27,78 \%)$.

Por outro lado, é preciso mencionar que dos valores indicados pelos 90 pares de enfermeiros na medida do diâmetro pupilar, somente 2 pares $(2,22 \%)$ apresentaram diferença superior a $1 \mathrm{~mm}$ na medida obtida do diâmetro pupilar de um ou ambos os olhos.

Há portanto indicação de que na aplicação do instrumento em teste poderá ocorrer frequientemente divergências de $1 \mathrm{~mm}$ nos valores apontados. Isto acarretará na série de avaliações seqüenciais do paciente, uma conduta semelhante àquela já mencionada, onde há menor valorização dessa diferença quando os valores encontram-se dentro dos parâmetros de normalidade, já que fisiológicamente estas variações ocorrem constantemente por diferentes estímulos simpáticos e parassimpáticos. Porém, quando as variações acontecerem indicando anisocoria ou fora dos valores considerados normais, serão motivos de avaliação mais frequientes e condutas mais direcionadas para dimensionar a alteração encontrada.

Os valores obtidos nas medidas dos diâmetros pupilares utilizando o cotejamento por pares de enfermeiros estão apresentados na Figura 1 .

Figura 1 - Reta de regressão linear mostrando os valores apontados pelos enfermeiros A e B conjuntamente.

Enfermeiro A

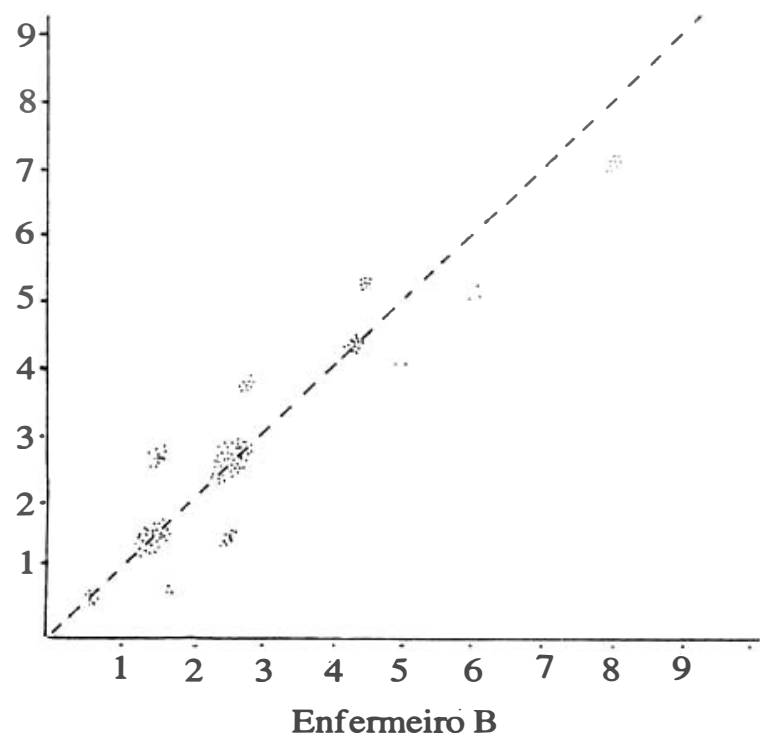

$$
\begin{aligned}
& r=0,905 \\
& a=0,260 \\
& b=0,923
\end{aligned}
$$

Resultados do teste " "t" observado " $t$ " crítico a $\quad 0,866 \quad 1,96$ b $-1,012 \quad 1,96$ 
A Figura 1 mostra uma maior concentração de pontos entre os valores de 2 a $4 \mathrm{~mm}$, indicando serem estas os valores mais apontados pelos enfermeiros, como resultado das medidas realizadas. Usualmente o diâmetro pupilar oscila entre 2,5 a $4,5 \mathrm{~mm}^{2}, 4$, justificando a maior incidência da indicação desses valores nos pacientes avaliados.

Os pontos encontrados na linha de identidade (linha pontilhada) são também mais freqüentes entre os valores 2 a $4 \mathrm{~mm}$, e são resultantes da coincidência dos valores indicados pelos enfermeiros " $A$ " $e$ " $B$ ".

Observando-se ainda a distribuição dos pontos no gráfico, nota-se que seu número na linha de identidade $\varepsilon$ maior do que fora dela, entre os valores $1 \mathrm{a} 3 \mathrm{~mm}$, quando se toma como referência tanto a linha abcissa como a ordenada. Esta relação inverte-se nos demais valores encontrados, demonstrando haver ocorrido pro- porcionalmente, maior número de coincidências nas medidas em que menores valores foram indicados.

Tais observações sugerem coerência com o resultado apresentado na Tabela 2 , onde nota-se que os erros nas medidas realizadas com o instrumento em teste, ocorrem mais freqüentemente nos círculos de tamanhos maiores.

Porem, ao aplicar-se aos valores a e b, obtidos no ajuste feito pela técnica de mínimos quadrados, o teste " $t$ ", concluiu-se que não hạ indicações, ao nível de $5 \%$ de desvios quer sistemáticos, quer proporcionais aos valores mensurados pelos dois grupos, ou seja, não há evidência estatística de que os pares de enfermeiros divergem nos valores que obtiveram.

Quanto ao uso da E.P., como mostra a Tabela 6 , nota-se que a grande maioria dos enfermeiros $(81,11 \%)$ não encontrou dificuldades ao medir o diâmetro pupilar.

Tabela 6. Distribuição dos enfermeiros, segundo indicação de dificuldades sentidas na verificação do diâmetro pupilar. São Paulo, 1988.

\begin{tabular}{c|c|c}
\hline Dificuldade & No & $\%$ \\
\hline Sim & 34 & 18,89 \\
Não & 1.46 & 81,11 \\
\hline Total & 180 & 100,00 \\
\hline
\end{tabular}

Tais resultados estão de acordo com autores como PASCOE ${ }^{16}$ que afirma que uma escala de ćŕrculos adaptada a uma espátula, é uma régua pupilar conveniente; e, LORD-FEROLI, MAGUIRE-McGINTY ${ }^{10}$, que testando comparativamente o uso de uma E.P., com a escala de círculos impressa em folha de registro, mostraram em seus resultados, que $\mathbf{9 8 \%}$ dos enfermei- ros que fizeram uso da espátula, reportaram-se satisfeitos com esta forma de medida.

Os 34 enfermeiros $(18,89 \%)$ que referiram sentir dificuldades ao verificar o diâmetro pupilar do paciente escolhido, especificaram 40 dificuldades encontradas, as quais foram categorizadas conforme sua natureza e estão apresentadas na Tabela 7.

Tabela 7. Tipo de dificuldades sentidas e indicadas por 34 dos enfermeiros participantes no estudo. São Paulo, 1988.

\begin{tabular}{l|c|c}
\hline Tipos de Dificuldades & No & $\%$ \\
\hline - consequientes a condiçóes desfavoráveis do paciente & 32 & 80,00 \\
- falta de experiência em usar o instrumento em teste & 4 & 10,00 \\
• diferença entre diâmetro dos círculos da espátula e pupila & 4 & 10,00 \\
\hline Total & 40 & 100,00 \\
\hline
\end{tabular}

$\mathrm{Na}$ Tabela 7 , nota-se que a maioria das dificuldades apontadas, $32(80,00 \%)$ foram consequientes às condições desfavoráveis do paciente.

A falta de experiência em executar a técnica com o instrumento em teste foi uma dificuldade apontada por 4 dos enfermeiros, embora os 180 enfermeiros participantes do estudo não tivessem experiência prévia em seu uso.

Considerando-se que todos os enfermeiros tiveram somente uma orientação previa, para utilizar a espátula em teste, pode-se concluir que a forma de utilizá-la é facilmente aprendida.

Nas dificuldades apontadas, percebe-se que a única inerente ao uso do instrumento testado, E a diferença entre os diâmetros dos ćŕrculos da espátula e da pupila do paciente. Esta dificuldade foi apontada por 4 enfermeiros, 2,22\% dos participantes do estudo, demonstrando uma baixa frequiência de situações, em que os usuários do instrumento apresentavam dúvidas quanto ao círculo da escala que melhor representa a grandeza das pupilas avaliadas.

Esta dificuldade pode, no entanto, ser sanada instruindo-se o usuário para registrar o valor medido entre dois dígitos, conforme a dúvida encontrada. Assim, no caso do enfermeiro estar indeciso se o dígito da escala que melhor representa a grandeza estudada é 5 ou 6 , registraria 
seu achado da seguinte forma: $>5$, < 6 . Esta forma de registro seria mais fidedigna com os valores achados e proporcionaria dados mais precisos para posteriores avaliações.

Finalizando, a E.P. testada demonstrou atender satisfatoriamente as características propostas a um instrumento para medida pupilar à beira do leito do paciente, pela confiabilidade, facilidade de confecção e baixo custo, inocuidade para o paciente e facilidade encontrada por parte do enfermeiro.

Assim sendo a E.P. testada pode ser considerada um importante instrumento que permite abandonar termos descritivos vagos e métodos subjetivos do exame pupilar, podendo-se assim adotar um padrão avaliativo mais seguro, principalmente para o atendimento de pacientes com risco de alteração da pressão intracraniana. Portanto, a E.P. é um valioso instrumento para utilização constante, quer no ensino, quer na assistência em unidades de emergência e cuidados intensivos e semi-intensivos, onde freqüentemente esses pacientes são encontrados.

\section{CONCLUSŌES}

1. Quanto à precisão da leitura de círculos milimetrados utilizando-se a E.P. verificou-se que:

- na quase totalidade dessas medidas, $97,23 \%$ e $95,02 \%$, os os resultados foram iguais aos dos verdadeiros valores da grandeza estudada;

- os erros predominaram nas medidas reali- zadas em fichas com círculos de diâmetros assimétricos e quando executadas em círculos de 5 ou mais milímetros de diâmetro ;

- o erro ocorreu na amplitude de $1 \mathrm{~mm}$, tanto para menor como maior do que o seu valor real.

2. Quanto à efetividade da medida do diâmetro pupilar com o uso da E.P., constatou-se que:

- $58,89 \%$ das medidas resultaram em valores coincidentes e $41,11 \%$ apontaram para valores discordantes, sendo que nestes predominou a diferença de $1 \mathrm{~mm}$.

- 45,55\% dos enfermeiros concordaram com seus pares no valor obtido na medida do diâmetro pupilar de ambos os olhos do paciente, $26,67 \%$ concordaram na medida de um dos olhos e $27,78 \%$ discordaram na medida de ambos os olhos ;

- mesmo aceitando a ocorrência de flutuações fisiológicas no diâmetro pupilar, não houve evidência estatística significativa a nível de $5 \%$ de que os pares de enfermeiros divergiram nos valores pupilares que obtiveram.

3. Quanto às dificuldades sentidas pelos enfermeiros ao utilizar a E.P., verificou-se que:

- a grande maioria dos enfermeiros $(81,11 \%)$ não indicou dificuldades na medida realizada e

- dos $18,89 \%$ enfermeiros que indicaram dificuldades, $80,00 \%$, foram consequientes às condições do paciente; $10,00 \%$ ocasionadas pela falta de experiência em usar o instrumento em teste e $10,00 \%$, devido a diferença entre o diâmetro dos círculos da espátula e da pupila medida.

\section{REFERÊNCIAS BIBLIOGRÁFICAS}

1 ANDERSON, M.S. Assessment under pressure: when your patient says, "my head hurts". Nursing, Springhouse, 14(9): 34-43, Sept. 1984.

2 ASSIS, J.L. de. Sistema motor ocular. In: CANELAS, H.M. et alii. Fisiopatologia do sistema nervoso. São Paulo: Savier, 1983. cap. 13, p. 195-243.

3 BORREGO, R.L., GARDNER, R.M. Classical conditioning of pupillary constriction. Percept. Mot. Skills, Missoula, 62(1): 315-22, Feb. 1986.

4 GONÇALVES, P. A pupila e suas alteraçōes estáticas e dinâmicas. In: - Oftabmologia. 4 ed, Rio de Janeiro: Atheneu, 1975. p. 387-401.

5 HICKEY, J:V. The neurological physical examination. In: The clinical practice of neurological and neurosurgical nur sing. 2. ed, Philadelphia: J.B. Lippincott, 1986. cap. 3, p. 59-89.

6 HINKLE, J. Nursing care of patients with minor head injury.J.-Neurosci. Nurs., Park Ridge, 20(1):8-16, Feb, 1988.

7 JOHNSON, L.K. If your patient has increased intracranial pressure, your goal should be: no surprise. Nursing, Springhouse, 13(6): 58-63, June 1983.
8 JONES, D.P., DEVERE, T.K. A simple objective pupillometer. J. Phys. (E), London, 11(8): 735-6, Aug. 1978.

9 KOIZUMI, M.S. Avaliação do nível de consciência em pacientes com traumatismo crâniano-encefálico. São Paulo, 1977. 56 p. (Dissertação de mestrado-Escola de Enfermagem da USP).

10 LORD-FEROLI, K., MAGUIRRE-McGINTY, M. Гoward a more objective approach to pupil assessment. $J$. Neurosurg, Nurs., Chicago, 17(5): 309-12, Oct. 1985.

11 MANCALL, E.L. Exame do sistema nervoso. In: $O$ exame neurologico. 2. ed, Rio de Janeiro: Colina, 1986. cap. 1 , p. 15-51.

12 METALIS, S.A., HESS, E.H. Pupillometric assessment of the readability of two video screen fonts. Percept.Mot. Skills, Missoula, 62(1): 279-82, Feb. 1986.

13 MILLER, M. Emergency management of the unconscious patient. Nurs. Clin. North Am., Philadelphia, 16(1): 59-73, Mar. 1981.

14 NAKAMAE, D.D., KOIZUMI, M.S. Exame clínico de enfermagem: Condiçōes físicas - parte III. Enf. Novas Dimens., São Paulo, 5(2): 10-6, mar./ab̆r. 1979. 
15 NEUROLOGIC care. Pensylvania, Springhouse, 1986. 183 p.

.16 PASCOE, D.A. A pupil gauge. Anaesthesia, London, 35(10): 1021, Oct. 1980.

17 PATTEN, J. As pupilas e suas reaçöes. In: Diagnóstico diferencial em neurologia. São Paulo: Roca, 1985. cap. 2, p. 3-10.

18 PLUM, F., POSNER, J.B. Diagnóstico de estupor e coma. 2. ed, Rio de Janeiro: Guanabara Koogan, 1977. p. 43-51.

18 PRESSMAN, M.R. et alii. Senile miosis: the possible contribution of disordered sleep and daytime sleepiness. $J$. Gerontol., Washington, 41(5): 629-34, Sept. 1986.

20 PRICE, M.B., DeVROOM, M.L. A quick and easy guide to neurological assessment. J.Neurosurg. Nurs., Chicago, 17(5): 313-20, Oct. 1985.

21 RICHER, F. et alii. Response selection and initiation in speeded reactions: a pupillometric analysis. J.Exp.Psy- chol., Washington, 9(3): 360-70, June 1983.

22 ROPPER, A.H. et alii. Computer-guided neurologic assessment in the neurologic intensive care unit. Heart Lung, Saint Louis, 1O(1): 54-60, Jan./Feb. 1981.

23 RUDY, E.B. Assessment of the neurological patient. In: . Advanced neurological and neurosurgical nursing. Saint Louis: Morby, 1984. cap. 2, p. 70-114.

24 SAVOY, S.M. The craniotomy patient: identifying the patient's neurological status. AORN J., Denver, 40(5): 716-24. Nov. 1984.

25 SCHLUROFF, M. Pupil responses to grammatical complexity of sentences. Brain Lang, New York, 17(1): 133-45, Sept. 1982.

26 UNIVERSIDADE DE SĀO PAULO. Instituto de Física. Elementos da teoria de erros. São Paulo, 1987. 44p.

27 WHI TNEY, F.W. Guidelines for neurological consultation. Nurse Pract., Trenton, 7(7): 13-8, July/Aug. 1982. 
“Espátula Pupilômetro” (E.P.)
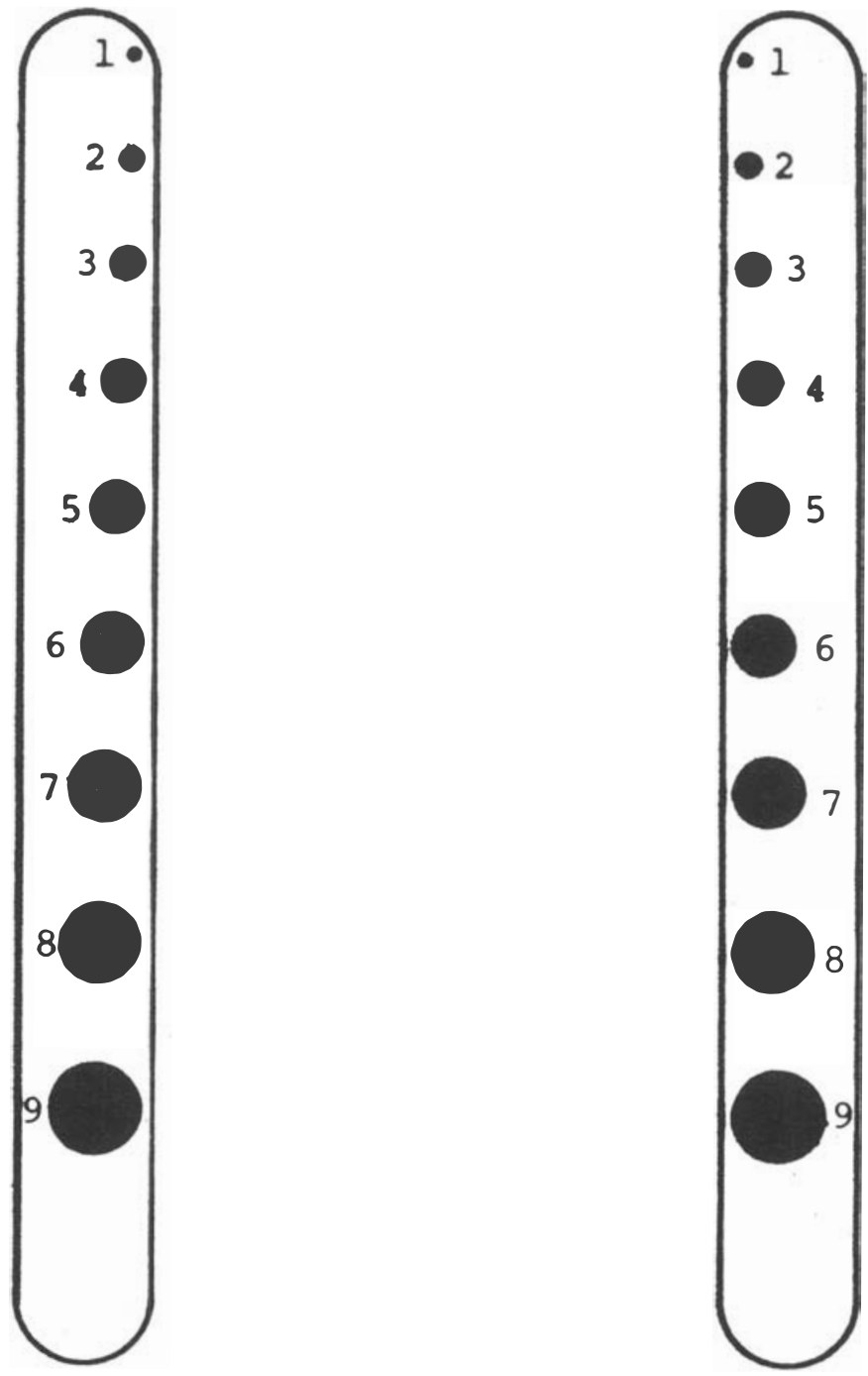

A. Face da E.P. para verificação do diâmetro pupilar do olho direito.
B. Face da E.P. para verificação do diâmetro pupilar do olho esquerdo. 
Anexo 2

Exemplo de "Ficha-Teste"

No은

Serie.

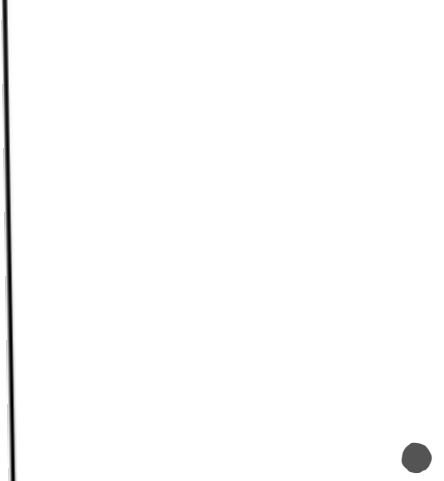

150 R. Bras. Enferm., Brasflia, 44 (2/3): 141-150, abril/set. 1991 